cession. It is, as I have already said, not this calcula. tion which is called in question by $\mathbb{M}$. Delaunay, but the fundamental idea.

M. Delaunay says the fluid will have precisely the same motion as the crust; and that, because the new motion of the crust is so slow. But it is clear that its s?owness has nothing to do with the matter. The fact is that the fluid and the crust not being connected together by any solid connection, no motion, whether small (i.e. slow) or not, can be suddenly communicated from the crust to the fluid mass. If the crust moved uniformly, as I have already said, and around a steady axis, the fluid might, after a lapse of ages, by friction and viscosity, acquire the motion of the crust. But if the crust is continually shifting from this steady position, however slowly, the fluid cannot suddenly acquire the new motion, and the crust slips over it ; and the thicker or thinner the crust, the greater or less is the solid mass to be shifted, and the less or the greater the precession produced, If the internal mass obeys at once the shifting motions of the crust, that mass cannot be fluid, but must be solid, and have a solid connection with the crust; in which case the whole question is yielded.

Mr. David Forbes speaks of the "Iabour" M. Delaunay has gone through in giving vent to his opinion. If the thing done is to be measured at all by the thing said, his labour must have been infinite; for what he has said is an impossibility. He has evidently altogether mistaken the problem. Mr. Hopkins's method stands unimpaired by his criticisms. Indeed Mr. Hopkins was not a man to advance a theory which could be apparently set aside by such slender means.

JOHN H. PRATT

\section{A THEORY OF A NERVOUS ATMOSPHERE}

UNDER the above title, Dr. Richardson, in a lecture published in the Medical Times and Gazette of last week, suggests a new theory in respect to nervous function. We propose in a few sentences to state simply the meaning of this theory.

The earlier physiological writers on the functions of the nervous system were under the impression that the brain, spinal cord, and other nervous centres acted after the manner of glands, and produced or secreted, as they said, a liquid. They called this assumed secreted liquid the nervous fluid, and they considered that it charges the nervous system, some also supposing that it makes even a circulation through tubular nervous channels or canals. It was not an uncommon notion that the nervous fluid conveys nourishment to the organs of the body; but the most common, and indeed generally accepted, hypothesis was, that it acts as a means of communication between all parts of the nervous system, and is the communicating medium of the impressions and motions derived from the outer world. Attempts were made to measure the rate of motion through this fuid, how long it took to convey an impression by it from brain to muscle.

The discovery of frictional electricity, the special discovery of the electric shock by Cuneus, of Leyden, in 1746, and the after discovery by Galvani of the inductive action of the prime conductor of the electrical machine on the muscles of frogs, threw quickly into the shade the speculations of the earlier neuro-physiologists. It was assumed at once that there exists a true animal electricity, that there is production of electricalaction within the bodies of all living animals, that there is conduction, and, in short, every mechanism and method for the carrying on, if we may so say, of electrical life. The discovery of the electrical organs of the torpedo, the dissection of the animal, the descriptions of its nerves by John Hunter, and the experiments made by a very earnest investigator, Mr. Walsh, aided greatly to establish the hypothesis which Galvani and his followers advanced, and which Volta, with the whole force of his experimental argument, failed to demolish.
Of late years the old hypothesis of the nervous fluid has been lost altogether, while the electrical hypotresis infinitely varied from its original and simple character, and infinitely varying with every new step of electrical discovery, has in a certsin sense retained its popular hold. It is true the hypothesis has rested on so much laboured obscurity that nobody has succeeded in making out of it a demonstration like the demonstration of the circulation of the blood, and no one has made it so simple that every scholar can read it when it is written, and every medical practitioner practise by it and act upon it as a known principle. It is true that since the time when Volta gave his undeniable proofs against the truth of the first inferences of Galvani, the best and most thoughtíl philosophers have felt doubts as to the electrical character of living action, and have looked on Galvani's construction of life as a beautiful crumbling ruin rather than as a temple befitting the worship of the gods of nature; and, lastly, it is true that whoever takes up to read the tomes or volumes of the most eminent writers on the subject of animal electricity is prone to lay them down again as he would the handles of a battery that master his will without appealing to his reason. All this is quite true; but still the electrical hypothesis has, as we before said, held its place; no attempt has been made to replace it; it has maintained around it a spell of fascination.

The theory that has been suggested by Dr. Richardson is in some sense a retum to the old view respecting nervous action, and in some sense also is an exterision to the nervous system of the physical idea of communication of motion by molecular disturbance. In a few words, the author of the theory supposes that the blood, as it circuIates in the vessels on which the structures of the body are constiucted, yields a diffusible vapour or atmosphere which charges the nervous system surrounding the molectules of nervous matter and pervading the whole nervous organism. He attempts to formulate the physical qualities of this vapour; it is probably an organic vapour containing carbon, hydrogen, and nitrogen; it is insoluble in blood, it is condensible by cold, diffusible by heat; it is retained after death longer in cold-blooded animals than in warm-blooded, and longer in warm-blooded animals that have died in cold than in those that have died in heat; it possesses conducting power, and as a physical substance is susceptible of variation of pressure; it connects the nervous system in all its parts together; it is the medium of communication during life between the outer and the inner existence; by the organs of the senses the impressions and motions derived from the outer worlcl are vibrated into or through the nervous atmosphere to the brain; in the living and healthy animal the nervous ether, if we may so designate it, is in correct tension, in the feeble it is diminished, in the dead it is absent or inactive; in the waking times of the living it is most active; it may be used up faster than it is produced during exercise; it is renewed during sleep.

On the supposition of the existence of a nervous ether or atmosphere as thus suggested, the author of the theory accounts for various phenomena connected with the partial or complete destruction of conscious, and even of organic life. The action of narcotic vapours is an illustration in point. It is assumed that these vapoursvapours of chloroform or alcohol, for example-taken into the blood and carried to the nervous system, become diffused through the nervous atmosphere, and by their presence interfere with its physical qualities and thus obscure function. "The foreign vapour that has been introduced benumbs; in other words, it interferes with the physical conduction of impressions through what should be the cloudless atmosphere between the outer and the inner existence."

Carrying out in a different way the same line of thought, the author of the theory to which we have 
specially called attention, accounts for the diffusion of some poisons through the body and for that rapid action of certain poisonous substances which so many experimenters have endeavoured, but not successfully, to explain; further, he suggests that in some instances poisonous products of decomposition generated within the body itself, in disease, may be diffused through the nervous ether, and that the sudden collapse of nervous function, which is often seen in acute disease, may be due to this cause. Finally, there may be conditions of disease in which there is unnatural tension of the nervous atmosphere, followed by disturbance of muscular motion, convulsion, or cerebral pressure, leading to apoplectic insensibility.

We have sketched out thus briefly the leading points of this theory of a nervous atmosphere or ether produced, during life, within and by the living organism, as a theory calculated to give rise to much discussion and device of new experiment.

\section{ASTRONOMICAL OBSERVATION}

\section{$\mathrm{T}$}

$\mathrm{E}$ statistics of modern astronomical observation would, we suspect, be very curious, if it were possible to get at them. A report showing the gradual increase in the number of telescopes manufactured during the last fifty years would be very interesting; and so would be a table comprising at once the advance in their dimensions and the diminution in their cost. The result would, we believe, be such as at first sight to cause great surprise among those unacquainted with the subject, or those whose recollection does not go back to days when five nches was as extraordinary an aperture for an objectglass, as double that size is now. But the value of these, as of other tabular statistics, would suffer material abatement, if they were applied to establish any other conclusions than those to which they directly lead. For instance they would probably be fallacious; if considered as inferring a proportionate increase in the number of important observations. In order to bring out such a result, we require, so to speak, another factor, and a very essential one-a corresponding increase in the number of competent observers. This, we fear, may not have been commensurate with the advance of optical means : at least, except upon the supposition of some such deficiency, it is difficult to understand what becomes of the multitude of really good object-glasses which are annually produced, not only in England, but in Germany and America. A large proportion of these, we are led to think, must be purchased to be looked at, and not looked through: or handled as mere toys for the amusement of people who do not know what to do with themselves in an idle evening. This was not so much the case in the early days of telescopemanufacture. The greatest master of figuring specula in his own time was also the greatest proficient in using them: it is needless to add the name of Sir William Herschel. And so the finest reflectors in Germany were placed at the same period in the hands of the leader of all accurate selenographical investigation, J. H. Schröter. These were "the right men in the right place." Even then, it may be said, many noble reflectors went, no one knows where, the greater part of them long before this time useless from tarnish, or, still more mortifying to think upon, ruined by unskilful repolishing. Still, admitting this, the disappearance of powerful instruments does not seem to have been so remarkable in those days as it is now, and the quantity of really valuable observations appears to have been greater in the end of the last and the early part of the present century, in proportion to the means of observing.

This is not a very encouraging view of the present state of this branch of astronomy. But, if well founded, as we believe it to be, we might expect that there would be some assignable reasons for it; and, in fact, several are sufficiently obvious. One certainly is, that the process of discovery is not, generally speaking, renewable. What has been once detected is usually placed on record, in bar of all future claims. So it has been in the science of music; a man might arise among us with the fervic genius of Handel, but he could not write the Hallelujah Chorus over again; and doubtless the spirit of Mendelssohn must have been cramped by the impossibility of employing many of the noblest and most impressive subjects wiich had been anticipated by his predecessors. And so it has been in the researches of geography. The enterprising explorer has now to go much farther in pursuit of "fre sh wcods and pastures new," and every Alpine season is so rapidly narrowing the number of summits untrodden by the foot of man, that the excitement of a first ascent will soon have to be sought in remoter regions. Thus in astronomy, though it cannot be said that there are no worlds left to conquer, yet all the larger and more conspicuous features of the heavenly bodies have been long ago so fully noted and recorded, that what remains for exploration is chiefly of that delicate character which, without being the less interesting from its minuteness, is less accessible, for that reason, to the possessors of ordinary instruments. And on this account many a student who might well have risen from the ranks in the earlier days of scientific campaigning, is now compeiled to remain in comparative obscurity-a mere spectator, when he might well have taken his place among the discoverer of fifty years ago.

Another reason why tools have multiplied without a corresponding increase of good work, may be this, that looking upon the observer and his instrument as a com plex apparatus, the improvement of the intelligent has not kept pace with that of the material part. In fact, it is impossible that it should. The eye is but what it was when David learned humility from considering God's heavens, the work of His fingers, the moon and the stars which $\mathrm{He}$ hath ordained; the intellect, though more developed and cultivated, is not more strong and piercing than it was in the days of Hipparchus; man does much more with his brain, but he has no more brain to do it with, than his uncivilised ancestors; and observers may, and will be, collectively multiplied without being indi vidually improved. Every man that has eyes does not know how to use them ; or, not failing in this respect, he may lack other requisites: he may not know what to look for, or where to find it; or he may be deficient in his handling of the faithful pencil or the expressive pen. And so it comes to pass that the capacities of instruments may be much in advance of the abilities of those who use them.

Besides all this, there is a physical obstacle of an entirely different character, which must not be forgotten; the unimprovable constitution of our own atmosphere. This will ever be a sore subject for the zealous observer, especially among ourselves. If even Secchi finds fault with the glorious Roman heavens, what have we not to regret in our own murky, and fuzzy, and restless skies? Who that has read the most graphic as well as instructive writings of Sir J. Herschel is likely to forget his complaints of "twitching; twirling, wrinkling, and horrible moulding?" and who that has had much actual experience of observatory work will not endorse all this with a very lively fellowfeeling? The nights may easily be numbered, during a long season, in which the defects of the atmosphere do not overlie those of the instrument, and when the observer has not rather to wish that he could see all that his telescope could show him, than to long for greater power or light, to be expended in making atmospheric disturbances yet more conspicuous and prejudicial. The only way to obviate this grievous hindrance is to get above it; and no man has yet done this except Professor Piazzi Smyth in 